\title{
Self-rated health and health-related quality of life among Chinese residents, China, 2010
}

\author{
Wen-lan Dong ${ }^{1,2} \mathbb{D}$, Yi-chong Li ${ }^{1}$, Zhuo-qun Wang ${ }^{1}$, Ying-ying Jiang ${ }^{1}$, Fan Mao ${ }^{1}$, Li Qi $^{1}$, Jian-qun Dong ${ }^{1 *}$
} and Li-min Wang ${ }^{1 *}$

\begin{abstract}
Background: Self-rated health (SRH) and health-related quality of life (HRQOL) are two outcome measures used to assess health status. However, little is known about population-based SRH and HRQOL in China.

Methods: Data from the 2010 China Chronic Disease and Risk Factor Surveillance, a nationally representative sample of 98,658 adults ( $\geq 18$-year-old) residing in China, were analyzed. SRH was assessed by asking "Would you say that, in general, your health is very good, good, general, poor, or very poor?" HRQOL was assessed by asking "For about how many days during the past 30 days was your health not good due to physical illnesses, injuries, or mental unhealthy?".

Results: Overall, $6.3 \%$ of participants rated their health as poor or very poor. The prevalence of poor/very poor health increased with advancing age ranging from $2.0 \%$ in the $18-24$ year-olds to $14.9 \%$ in those $\geq 75$ years-old, while it decreased with education levels from $13.0 \%$ in illiterates/those with some primary school education to 2.2 $\%$ in college graduates or above. Additionally, women were more likely than men to rate their health as poor or very poor (7.2\% vs. $5.4 \%)$. The reported rate of poor/very poor health was higher in western region residents compared to those in the east (7.4\% vs. $5.3 \%)$. The mean numbers of self-reported physically unhealthy days, injury-caused unhealthy days, or mentally unhealthy days during the past 30 days were 1.48, 0.20, and 0.54, respectively. Older adults had more physically unhealthy days than the younger ones ranging from 2.92 days in those $\geq 75$ year-old to 0.95 days in 18-24 year-olds. Women had more physically unhealthy days and mentally unhealthy days than men (1.72 vs. 1.23; 0.62 vs. 0.46 , respectively). The highest mean number of physically unhealthy days (2.32) was reported by illiterates or those with some primary school education. The highest mean number of mentally unhealthy days (0.86) reported by college graduates or above.
\end{abstract}

Conclusions: Substantial variations existed in SRH and HRQOL among age groups, gender groups, education groups, and across regions in China. Considering these disparities will be important when developing health policies and allocating resources.

Keywords: Self-rated health, Health-related quality of life, Public health

\footnotetext{
* Correspondence: workdong@163.com; wlm65@126.com

${ }^{1}$ National Center for Chronic and Noncommunicable Disease Control and Prevention, Beijing 100050, People's Republic of China

Full list of author information is available at the end of the article
}

\section{Ciomed Central}

(C) 2016 Dong et al. Open Access This article is distributed under the terms of the Creative Commons Attribution 4.0 International License (http://creativecommons.org/licenses/by/4.0/), which permits unrestricted use, distribution, and reproduction in any medium, provided you give appropriate credit to the original author(s) and the source, provide a link to the Creative Commons license, and indicate if changes were made. The Creative Commons Public Domain Dedication waiver (http://creativecommons.org/publicdomain/zero/1.0/) applies to the data made available in this article, unless otherwise stated. 


\section{Background}

The World Health Organization (WHO) has defined healthiness as "a state of complete physical, mental, and social well-being and not merely the absence of disease or infirmity" [1]. Health is seen by the public health community as a multidimensional construct that includes physical, mental, and social domains [2]. Selfrated health $(\mathrm{SRH})$ and health-related quality of life (HRQOL) are two outcome measures used to assess health status. These two measures are self-reported, inexpensive, and easy to use.

SRH is easily measured in large population surveys, and is a useful "opener" in interview situations that allow interviewers to seek more nuanced and complex responses about people's perceptions of their health [3]. Poor SRH status has also been shown to be independently predictive of subsequent morbidity and higher health care utilization $[4,5]$. The concept of health-related quality of life is that an individual's or group's perceived physical and mental health over time [6]. On the individual level, HRQOL includes physical and mental health perceptions and their correlates, including health risks and conditions, functional status, social support, and socioeconomic status [7-9]. On the community level, HRQOL includes resources, conditions, policies, and practices that influence a population's health perceptions and functional status [10]. HRQOL questionnaires on perceived physical and mental health and function have become an important component of health surveillance and are generally considered valid indicators of service needs and intervention outcomes [6].

Measuring SRH and HRQOL through continual surveillance would identify health disparities, track population trends, and provide the public's perspective to guide health policies $[6,11]$. SRH and HRQOL have been tracked in many countries. For instance, in the early 1990s, the Centers for Disease Control and Prevention (CDC) in the U.S. developed and validated a brief set of questions to track SRH and HRQOL in states and communities. From 1993 through 2001, more than 1.2 million adults aged 18 years old or above in the U.S. answered these questions on the population-based Behavioral Risk Factor Surveillance survey (BRFSS) [6, 11]. SRH and HRQOL have also been being tracked through National Health Survey (NHS) in Candia, Australian, French, Xincarpor, et al. [3, 12-14].

However, there is limited information regarding the SRH and HRQOL status in China. The aim of this study was to estimate status of SRH and HRQOL for Chinese adults based on the nationally-representative data from China Chronic Disease and Risk Factor Surveillance (CCDRFS) in 2010. We hypothesize that both SRH and HRQOL will differ among subgroups such as age, gender, education, marital status, residence location (urban/rural), and geographic location groups. Based on the findings from this study, we discussed the implications of the SHR and HRQOL on developing health policies in China.

\section{Methods \\ Surveillance and study sample}

The CCDRFS is an ongoing, nationally representative surveillance survey administered by China's National Center for Chronic and Noncommunicable Disease Control and Prevention. The 2010 CCDRFS was carried out from August to November in 2010 using the national disease surveillance points system, which encompassed 162 districts/counties from all 31 provinces, autonomous regions and municipalities in the mainland China. The establishment, history, and degree of representativeness of the national disease surveillance points system were explained elsewhere $[15,16]$.

The 2010 CCDRFS was approved by the ethics committee of the Chinese Center for Disease Control and Prevention and the written informed consent forms were obtained from each participant before data collection. The 2010 CCDRFS was conducted by gathering participants in certain central locations. The data were collected in face-to-face interviews on SRH and HRQOL questionnaires. All investigators participated in national or provincial training courses and were qualified to engage in field activities after passing standard exams. Detailed information on quality control and the data analyses in the 2010 CCDRFS has been published elsewhere [17].

Multistage stratified cluster sampling was used to select participants for the 2010 CCDRFS. In the first stage of sampling, four townships were randomly sampled from each surveillance district/county using the method of probability proportional to size. Three villages or residential areas were then sampled from each chosen township using the same method as in the previous stage. Subsequently, a residential group (at least 50 families) was sampled from each chosen village or residential area by simple random sampling. Finally, an individual of at least 18 years old was sampled in each family by means of a Kish grid. About $9.4 \%$ of the sampled families could not be located after three attempts and these households were replaced by others with similar family structures. A total of 98658 interviews were included in the final analyses [18].

\section{Measures}

SRH and HRQOL were assessed based on the participants' answers for the following four questions: 
1. Would you say that, in general, your health is very good, good, general (not good/not poor), poor, or very poor? [SRH]

2. Now thinking about your physical illness, for how many days during the past 30 days was your health not good due to physical illness? [Physically unhealthy days]

3. Now thinking about your injury, which includes accident injury or intentional damage such as fall, traffic, etc., for how many days during the past 30 days was your health not good due to injury? [Injury-caused unhealthy days]

4. Now thinking about your mental health, which includes stress, depression, and problems with emotions, for how many days during the past 30 days was your health not good due to mental unhealthy? [Mentally unhealthy days]

We obtained data based on demographic characteristics (age, gender, and marital status), socioeconomic status (educational level), residence location (rural/urban), and geographic region of China. The corresponding subcategories were shown in Table 1 . We calculated 1) the percentages and $95 \%$ confidence interval $[\mathrm{CI}]$ for respondents reporting very good, good, general, poor, and very poor based on the SRH questions and 2) the mean number and $95 \% \mathrm{CI}$ for the physically unhealthy days, injury-caused unhealthy days, and mentally unhealthy days based on HRQOL questions [19].

\section{Statistical analysis}

In the present study, weighting was conducted in all statistical analyses to obtain nationally representative estimates. The weights were the products of sampling selection weight, which was the reciprocal of the probability of a particular individual being selected, and a post-stratification factor that adjusted for age, rural/ urban residence, and geographic location in accordance with the 2009 Chinese population estimates obtained from the National Bureau of Statistics of China [20].

We first determined the sample characteristics and then estimated the percent of SRH and the mean number of physically, injury-caused, and mentally unhealthy days on the basis of a specific characteristic of population, such as age, gender, marital status, education, rural/urban residence, and geographic location. Age was divided into 7 groups: $18-24,25-34,35-44,45-54,55-$ 64, 65-74, and 75+. Marital status was classified into 3 categories: single, married/cohabiting, and separated/divorced/widowed/others. Socioeconomic status was assessed based on the education levels that was categorized as 5 groups: illiterate or some primary school, primary school graduate or some junior high school, junior high school graduate or some senior high school, senior
Table 1 Characteristics of the study sample of the 2010 CCDRFS survey

\begin{tabular}{|c|c|c|c|}
\hline \multirow[t]{2}{*}{ Characteristic } & \multicolumn{3}{|c|}{ Respondents $(n=98658)$} \\
\hline & No. & (\%) & Weighted ${ }^{\text {a }} \%$ \\
\hline \multicolumn{4}{|l|}{ Age group (years) } \\
\hline $18-24$ & 8372 & 8.5 & 16.1 \\
\hline $25-34$ & 13534 & 13.7 & 18.2 \\
\hline $35-44$ & 23271 & 23.6 & 23.3 \\
\hline $45-54$ & 22837 & 23.2 & 18.2 \\
\hline $55-64$ & 18362 & 18.6 & 13.6 \\
\hline $65-74$ & 8902 & 9.0 & 7.0 \\
\hline$\geq 75$ & 3380 & 3.4 & 3.6 \\
\hline \multicolumn{4}{|l|}{ Gender } \\
\hline Man & 45143 & 45.8 & 50.8 \\
\hline Woman & 53515 & 54.2 & 49.2 \\
\hline \multicolumn{4}{|l|}{ Education } \\
\hline Illiterate or some primary school & 24108 & 24.4 & 19.5 \\
\hline $\begin{array}{l}\text { Primary school graduate or some } \\
\text { junior high school }\end{array}$ & 18965 & 19.2 & 18.2 \\
\hline $\begin{array}{l}\text { Junior high school graduate or some } \\
\text { senior high school }\end{array}$ & 31378 & 31.8 & 35.8 \\
\hline $\begin{array}{l}\text { Senior high school graduate or some } \\
\text { college }\end{array}$ & 16045 & 16.3 & 17.5 \\
\hline College graduate or above & 8162 & 8.3 & 9.0 \\
\hline \multicolumn{4}{|l|}{ Marital status } \\
\hline Single & 8432 & 8.5 & 8.3 \\
\hline Married or cohabiting & 80193 & 81.3 & 78.0 \\
\hline Separated/divorced/widowed/others & 10033 & 10.2 & 13.7 \\
\hline \multicolumn{4}{|l|}{ Place of residence } \\
\hline Urban & 38928 & 39.5 & 31.2 \\
\hline Rural & 59730 & 60.5 & 68.8 \\
\hline \multicolumn{4}{|l|}{ Geographic location } \\
\hline Eastern China & 32965 & 33.4 & 39.9 \\
\hline Central China & 30569 & 31.0 & 32.5 \\
\hline Western China & 35124 & 35.6 & 27.6 \\
\hline Total & 98658 & 100.0 & 100.0 \\
\hline
\end{tabular}

a Complex weights were used to obtain nationally representative estimates Source: Data from the 2010 China Chronic Disease and Risk Factor Surveillance survey (CCDRFS)

high school graduate or some college, and college graduate or above. The country was divided by geography: eastern, central, and western regions according to the National Bureau of Statistics. The eastern region includes Beijing, Tianjin, Hebei, Liaoning, Shanghai, Jiangsu, Zhejiang, Fujian, Shandong, Guangdong, and Hainan. The central region includes Shanxi, Jilin, Heilongjiang, Anhui, Jiangxi, Henan, Hubei, and Hunan. The western region includes Inner Mongolia, Guangxi, 
Chongqing, Sichuan, Guizhou, Yunnan, Tibet, Shaanxi, Gansu, Qinghai, Ningxia, and Xinjiang.

In Table 2, We combined the categories poor and very poor because the percentage of very poor is very low and conducted Rao-Scott $\chi^{2}$ tests for percentages of SRH (very good, good, general, and poor/very poor) to test the differences in proportion by categories.

In Table 3, we used CIs to compare the mean number of physically unhealthy days, injury-caused unhealthy days, and mentally unhealthy days by categories. If the $95 \%$ CIs of these estimates did not overlap, these estimates were considered different from each other at the $\alpha=0.05$ significance level $[21,22]$.

In Table 4, we examined 1) the independent effects of covariates on SRH (very good, good, general, poor/very poor) by modeling a multiple ordered logistic regression and 2) the independent effects of covariates on unhealthy days by multiple liner regression. The age, gender, marital status, education, rural/urban residence, and geographic location were independent variables. Logistic model was presented as odds ratios (OR) with $95 \% \mathrm{CI}$ and liner regression model was presented as $\beta$ with $P$ value. These ordered categorical variables were tested as a continuous variable in a logistic regression model [23]. The respondents who reported "don't know/not sure" or "refused" were excluded from the analysis. All statistical analysis were performed using SAS version 9.3 (SAS Institute Inc., Cary, USA) and CIs were estimated while accounting for complex sample design using the Taylor's series method [24] with finite population correction.

\section{Results}

\section{Characteristics of the respondents}

The sample characteristics are shown in Table 1. The majority of the respondents were between 35 and 54 years old $(46.8 \%)$. There were more women than men (54.2\% vs. $45.8 \%)$. About one third (31.8 \%) were junior high school graduates or some high school. 81.3 \% were either married or cohabiting. $60.5 \%$ lived in rural areas and $35.6 \%$ lived in western China. The Table 1 also shows the distributions of the various characteristics after weighting for obtaining nationally representative estimates.

\section{Distribution of SRH}

Table 2 shows the distribution of SRH among Chinese adults aged $\geq 18$ years in 2010 . Overall, $9.7 \%$ rated their health as being very good, $47.9 \%$ as good, $36.2 \%$ as general, and only $6.3 \%$ as poor or very poor. The proportion of SRH was significantly different among age groups $\left(X^{2}=21.07, P<0.01\right)$, gender groups $\left(X^{2}=60.17\right.$, $P<0.01)$, education groups $\left(\chi^{2}=68.03, P<0.01\right)$, marital status groups $\left(X^{2}=72.14, P<0.01\right)$, urban-rural group $\left(\chi^{2}=2.98, P<0.05\right)$, and geographic location $\left(\chi^{2}=4.35\right.$, $P<0.01)$.

\section{Distribution of HRQOL}

Table 3 shows the mean number of physically unhealthy days, injury-caused unhealthy days, and mentally unhealthy days during the past 30 days among Chinese adults aged $\geq 18$ years.

\section{Physically unhealthy days}

Adults reported that they had an average of 1.48 physically unhealthy days during the past 30 days. The physically unhealthy days increased modestly with advancing age ranging from 0.95 days in 18-24 years old age group to 2.92 days in 75 years old age group. Woman reported more physically unhealthy days than man (1.72 vs. 1.23$)$. The adults with less education reported more physically unhealthy days compared to those with more education ranging from 2.32 days with illiterate or some primary school to 1.17 days with college graduate or above. The highest physically unhealthy days were reported in the adults who were separated, divorced, widowed, or in other marital status groups (2.24 days).

\section{Injury-caused unhealthy days}

Adults reported that they had an average of 0.20 (95\% CI: 0.17-0.24) injury-caused days during the past 30 days. The differences of mean injury days were not found to be significant among age groups, gender groups, education groups, marital status groups, urban-rural groups, and geographic location groups.

\section{Mentally unhealthy days}

Adults reported that they suffered an average number of 0.54 mentally unhealthy days during the past 30 days. Women reported more mentally unhealthy days than men $(0.62$ vs. 0.46$)$. The adults with more education reported more mentally unhealthy days than those with less education. The mentally unhealthy days decreased from 0.86 days with college graduates or above to 0.50 days with illiterate or some primary school. There were no significant differences of mentally unhealthy days among age groups, marital status groups, urbanrural groups, and geographic location groups.

\section{Associated factors with SRH and HRQOL}

Table 4 shows the independent effect of various covariates on the SRH by ordinal logistic regression and unhealthy days by liner regression at the individual level as indicated.

Age, gender, education, and geographic location were independently associated with the SRH. For instance, in adults aged 75 years or older, the cumulative odds of reporting the poorer health 4.88 times higher than those 
Table 2 Weight percent of self-rated health among Chinese adults aged $\geq 18$ years, China, 2010 ( $n=98658$ missing =20)

\begin{tabular}{|c|c|c|c|c|c|c|c|c|c|}
\hline \multirow[t]{2}{*}{ Characteristic } & \multirow[b]{2}{*}{$\begin{array}{l}\text { Number of } \\
\text { respondents }\end{array}$} & \multicolumn{2}{|l|}{ Very good } & \multicolumn{2}{|l|}{ Good } & \multicolumn{2}{|l|}{ General } & \multirow{2}{*}{$\begin{array}{l}\text { PoorNery poor } \\
\text { Weighted percent } \\
(95 \% \text { Cl) }\end{array}$} & \multirow[t]{2}{*}{$p$} \\
\hline & & $\begin{array}{l}\text { Weighted percent } \\
(95 \% \mathrm{Cl})\end{array}$ & $\begin{array}{l}\text { Number of } \\
\text { respondents }\end{array}$ & $\begin{array}{l}\text { Weighted percent } \\
(95 \% \mathrm{Cl})\end{array}$ & $\begin{array}{l}\text { Number of } \\
\text { respondents }\end{array}$ & $\begin{array}{l}\text { Weighted percent } \\
(95 \% \mathrm{Cl})\end{array}$ & $\begin{array}{l}\text { Number of } \\
\text { respondents }\end{array}$ & & \\
\hline \multicolumn{10}{|l|}{ Age group (years) } \\
\hline $18-24$ & 1405 & $18.5(15.5-21.5)$ & 4693 & $55.4(52.2-58.6)$ & 2109 & $24.1(21.1-27.1)$ & 165 & $2.0(1.6-2.5)$ & \multirow[t]{7}{*}{$P<0.01$} \\
\hline 25-34 & 1546 & $13.1(11.2-15.1)$ & 7633 & $55.1(52.7-57.4)$ & 3967 & $29.1(26.6-31.5)$ & 387 & $2.7(2.3-3.3)$ & \\
\hline $35-44$ & 1805 & $9.4(7.9-11.0)$ & 12205 & $51.4(49.1-53.7)$ & 8092 & $34.4(32.3-36.5)$ & 1165 & $4.8(4.2-5.5)$ & \\
\hline $45-54$ & 1146 & $6.3(5.1-7.5)$ & 10603 & $45.5(43.7-47.4)$ & 9272 & $40.5(38.4-42.6)$ & 1809 & 7.7 (7.0-8.4) & \\
\hline $55-64$ & 698 & $4.4(3.6-5.3)$ & 7274 & 38.8 (36.8-40.8) & 8426 & $46.1(44.0-48.2)$ & 1961 & $10.6(9.7-11.7)$ & \\
\hline $65-74$ & 228 & $3.0(2.3-3.6)$ & 3028 & 33.5 (31.6-35.3) & 4410 & $49.3(47.8-50.9)$ & 1232 & $14.2(12.7-16.0)$ & \\
\hline$\geq 75$ & 92 & $2.9(2.1-3.8)$ & 1033 & $30.0(27.7-32.3)$ & 1766 & $52.2(48.9-55.4)$ & 488 & $14.9(12.6-17.4)$ & \\
\hline \multicolumn{10}{|l|}{ Gender } \\
\hline Man & 3737 & $11.1(9.6-12.6)$ & 22136 & $49.4(47.5-51.2)$ & 16437 & $34.1(32.4-35.8)$ & 2827 & $5.4(4.9-6.0)$ & \multirow[t]{2}{*}{$P<0.01$} \\
\hline Woman & 3183 & $8.1(6.9-9.4)$ & 24333 & $46.3(44.6-48.1)$ & 21605 & $38.3(36.6-40.0)$ & 4380 & $7.2(6.5-8.0)$ & \\
\hline \multicolumn{10}{|l|}{ Education } \\
\hline Illiterate or some primary school & 813 & $4.2(3.4-5.2)$ & 9962 & $39.3(37.0-41.6)$ & 10269 & $43.5(41.5-45.6)$ & 3054 & $13.0(11.7-14.4)$ & \multirow[t]{5}{*}{$P<0.01$} \\
\hline $\begin{array}{l}\text { Primary school graduate /some } \\
\text { junior high school }\end{array}$ & 1021 & $6.6(5.2-8.4)$ & 8839 & $46.6(44.2-49.1)$ & 7512 & $38.9(36.4-41.4)$ & 1590 & $7.9(7.1-8.8)$ & \\
\hline $\begin{array}{l}\text { Junior high school graduate/some } \\
\text { senior high school }\end{array}$ & 2765 & $11.6(9.8-13.8)$ & 15849 & $51.4(48.9-53.9)$ & 11121 & $32.8(30.6-35.1)$ & 1637 & $4.2(3.7-4.8)$ & \\
\hline $\begin{array}{l}\text { Senior high school graduate/some } \\
\text { college }\end{array}$ & 1417 & $12.3(10.7-13.9)$ & 7814 & $50.1(48.0-52.2)$ & 6111 & $34.0(31.6-36.4)$ & 703 & $3.7(3.2-4.2)$ & \\
\hline College graduate or above & 904 & $14.5(12.7-16.5)$ & 4005 & $50.8(48.5-53.1)$ & 3029 & $32.5(29.6-35.6)$ & 223 & $2.2(1.8-2.7)$ & \\
\hline \multicolumn{10}{|l|}{ Marital status } \\
\hline Single & 1323 & $18.215 .7-21.03)$ & 4510 & $53.6(50.5-56.6)$ & 2307 & $25.4(22.5-28.4)$ & 291 & $2.9(2.3-3.6)$ & \multirow[t]{3}{*}{$P<0.01$} \\
\hline Married or cohabiting & 5247 & 8.7(7.4-10.2) & 37903 & $47.7(45.9-49.5)$ & 31259 & $37.3(35.5-39.2)$ & 5767 & $6.35 .7-7.0)$ & \\
\hline Separated/divorced/widowed/others & 350 & $4.3(3.5-5.3)$ & 4056 & 40.5(36.9-44.1) & 4476 & $43.3(40.5-46.2)$ & 1149 & 11.8(10.3-13.6) & \\
\hline \multicolumn{10}{|l|}{ Place of residence } \\
\hline Urban & 3029 & $9.8(8.5-11.1)$ & 17371 & $45.2(42.8-47.6)$ & 16129 & $39.3(36.8-41.8)$ & 2392 & $5.7(4.7-6.8)$ & \multirow[t]{2}{*}{$P<0.05$} \\
\hline Rural & 3891 & $9.6(7.7-11.4)$ & 29098 & $49.1(46.9-51.3)$ & 21913 & $34.7(32.6-36.9)$ & 4815 & $6.6(5.9-7.4)$ & \\
\hline
\end{tabular}


Table 2 Weight percent of self-rated health among Chinese adults aged $\geq 18$ years, China, 2010 ( $n=98658$ missing =20) (Continued)

\begin{tabular}{|c|c|c|c|c|c|c|c|c|c|}
\hline \multicolumn{10}{|c|}{ Geographic location } \\
\hline Eastern China & 3137 & $13.0(10.8-15.1)$ & 14938 & $47.1(44.6-49.6)$ & 12748 & $34.6(32.3-36.9)$ & 2134 & $5.3(4.5-6.2)$ & \multirow[t]{4}{*}{$P<0.01$} \\
\hline Central China & 1877 & $7.3(6.1-8.4)$ & 14185 & $48.4(44.8-51.9)$ & 12130 & $37.8(34.5-41.0)$ & 2376 & $6.6(5.6-7.8)$ & \\
\hline Western China & 1906 & $7.6(4.2-10.9)$ & 17346 & $48.5(45.6-51.4)$ & 13164 & $36.5(33.2-39.8)$ & 2697 & $7.4(6.3-8.7)$ & \\
\hline Total & 6920 & $9.7(8.3-10.9)$ & 46469 & $47.9(46.2-49.6)$ & 38042 & $36.2(34.5-37.8)$ & 7207 & $6.3(5.7-6.9)$ & \\
\hline
\end{tabular}

Note: Percents were weighted to represent the total population of the national disease surveillance points system with post-stratification for age and gender.

The number in parentheses is the percent of the $95 \%$ confidence interval which taking into account the complex survey design.

Source: Data from the 2010 China Chronic Disease and Risk Factor Surveillance survey 
Table 3 Mean number unhealthy days during the past 30 days among Chinese adults aged $\geq 18$ years, China, 2010 $(n=98658)$

\begin{tabular}{|c|c|c|c|c|c|c|}
\hline \multirow[t]{2}{*}{ Characteristic } & \multicolumn{2}{|c|}{ Physically unhealthy days ${ }^{a}$} & \multicolumn{2}{|c|}{ Injury-caused unhealthy days ${ }^{b}$} & \multicolumn{2}{|c|}{ Mentally unhealthy days ${ }^{c}$} \\
\hline & $\begin{array}{l}\text { Number of } \\
\text { respondents }\end{array}$ & $\begin{array}{l}\text { Mean number of } \\
\text { days ( } 95 \% \mathrm{Cl} \text { ) }\end{array}$ & $\begin{array}{l}\text { Number of } \\
\text { respondents }\end{array}$ & $\begin{array}{l}\text { Mean number of } \\
\text { days }(95 \% \mathrm{Cl}\end{array}$ & $\begin{array}{l}\text { Number of } \\
\text { respondents }\end{array}$ & $\begin{array}{l}\text { Mean number of } \\
\text { days ( } 95 \% \mathrm{Cl}\end{array}$ \\
\hline \multicolumn{7}{|l|}{ Age group (years) } \\
\hline $18-24$ & 7530 & $0.95(0.79-1.10)$ & 7548 & $0.26(0.17-0.35)$ & 7553 & $0.58(0.45-0.71)$ \\
\hline $25-34$ & 12106 & $0.93(0.81-1.05)$ & 12109 & $0.20(0.13-0.26)$ & 12074 & $0.55(0.46-0.65)$ \\
\hline $35-44$ & 21058 & $1.15(1.04-1.27)$ & 21079 & $0.15(0.11-0.19)$ & 21009 & $0.50(0.43-0.57)$ \\
\hline $45-54$ & 20522 & $1.70(1.51-1.88)$ & 20700 & $0.23(0.19-0.27)$ & 20607 & $0.57(0.48-0.65)$ \\
\hline $55-64$ & 16385 & $2.12(1.93-2.31)$ & 16628 & $0.22(0.18-0.26)$ & 16545 & $0.56(0.49-0.63)$ \\
\hline $65-74$ & 7813 & $2.74(2.48-3.01)$ & 7999 & $0.20(0.14-0.26)$ & 7949 & $0.49(0.40-0.57)$ \\
\hline$\geq 75$ & 2861 & $2.92(2.42-3.43)$ & 2948 & $0.21(0.13-0.28)$ & 2930 & $0.41(0.31-0.51)$ \\
\hline \multicolumn{7}{|l|}{ Gender } \\
\hline Man & 40279 & $1.23(1.12-1.34)$ & 40572 & $0.23(0.18-0.28)$ & 40481 & $0.46(0.40-0.53)$ \\
\hline Woman & 47996 & $1.72(1.56-1.88)$ & 48439 & $0.17(0.14-0.21)$ & 48186 & $0.62(0.54-0.70)$ \\
\hline \multicolumn{7}{|l|}{ Education } \\
\hline Illiterate or some primary school & 7404 & $2.32(2.12-2.52)$ & 7476 & $0.22(0.17-0.27)$ & 7465 & $0.50(0.43-0.58)$ \\
\hline Primary school graduate/some junior high school & 14658 & $1.66(1.47-1.84)$ & 14773 & $0.24(0.15-0.32)$ & 14754 & $0.51(0.41-0.60)$ \\
\hline $\begin{array}{l}\text { Junior high school graduate/some senior high } \\
\text { school }\end{array}$ & 28476 & $1.12(1.00-1.23)$ & 28652 & $0.21(0.16-0.25)$ & 28605 & $0.45(0.37-0.53)$ \\
\hline Senior high school graduate/some college & 16851 & $1.27(1.09-1.46)$ & 17025 & $0.19(0.14-0.25)$ & 16938 & $0.62(0.53-0.72)$ \\
\hline College graduate or above & 20886 & $1.17(0.99-1.34)$ & 21085 & $0.13(0.09-0.18)$ & 20905 & $0.86(0.72-1.00)$ \\
\hline \multicolumn{7}{|l|}{ Marital status } \\
\hline Single & 7618 & $1.07(0.93-1.21)$ & 7624 & $0.26(0.16-0.36)$ & 7629 & $0.65(0.52-0.78)$ \\
\hline Married or cohabiting & 71647 & $1.47(1.34-1.59)$ & 72233 & $0.20(0.16-0.24)$ & 71923 & $0.51(0.44-0.58)$ \\
\hline Separated/divorced/widowed/others & 9010 & $2.24(1.90-2.57)$ & 9154 & $0.20(0.14-0.27)$ & 9115 & $0.66(0.53-0.78)$ \\
\hline \multicolumn{7}{|l|}{ Place of residence } \\
\hline Urban & 35561 & $1.57(1.37-1.78)$ & 36182 & $0.16(0.14-0.19)$ & 36093 & $0.65(0.55-0.74)$ \\
\hline Rural & 52714 & $1.43(1.27-1.59)$ & 52829 & $0.22(0.17-0.28)$ & 52574 & $0.49(0.40-0.58)$ \\
\hline \multicolumn{7}{|l|}{ Geographic location } \\
\hline Eastern China & 29155 & $1.43(1.23-1.63)$ & 29398 & $0.18(0.14-0.21)$ & 29341 & $0.51(0.40-0.61)$ \\
\hline Central China & 28048 & $1.30(1.08-1.51)$ & 28444 & $0.16(0.11-0.21)$ & 28247 & $0.49(0.37-0.61)$ \\
\hline Western China & 31072 & $1.76(1.50-2.01)$ & 31169 & $0.30(0.18-0.42)$ & 31079 & $0.64(0.49-0.79)$ \\
\hline Total & 88275 & $1.48(1.35-1.60)$ & 89011 & $0.20(0.17-0.24)$ & 88667 & $0.54(0.47-0.61)$ \\
\hline
\end{tabular}

${ }^{a}$ Number of respondents to physically unhealthy day $=88275$, missing $=10383$

${ }^{b}$ Number of respondents to injury-caused unhealthy days $=89011$, missing $=9647$

${ }^{c}$ Number of respondents to mental unhealthy $=88667$, missing $=9991$

Note: The mean numbers of days were weighted to represent the total population of the national disease surveillance points system with post-stratification for age and gender; The number in parentheses is the mean number of days of the $95 \%$ confidence interval which taking into account the complex survey design.

Source: Data from the 2010 China Chronic Disease and Risk Factor Surveillance survey

in $18-24$ years old. The cumulative odds increased steadily with increasing age and declined steadily with educational levels. Women were 1.25 times more likely to rate poorer health, than men. The adults in the central region were 1.42 times and in the western region were 1.37 times higher than those in the eastern region.

Age, gender, education, and geographic location were independently associated with the physical unhealthy days. For instance, in adults aged 75 years or older, reporting unhealthy days increase 1.78 days than those in $18-24$ years old. $\beta$ value increased steadily with increasing age and declined steadily with educational levels. Women reported more physically unhealthy days (0.47) than man. Adults in the west region also reported more physically unhealthy days $(0.32)$ than those in the eastern region.

Gender and education were independently associated with the mentally unhealthy days. Women reported more mentally unhealthy days (1.37) than man. Adults with senior high school graduate and college graduate 
Table 4 Independent effects of covariates on self-rated health and unhealthy days in individuals, China, 2010

\begin{tabular}{|c|c|c|c|c|c|c|c|}
\hline \multirow[b]{2}{*}{ Characteristic Cumulative OR ${ }^{\mathrm{b}}\left(95 \% \mathrm{Cl}^{\mathrm{a}}\right)$} & \multirow{2}{*}{$\begin{array}{l}\text { Self-rated health }^{a} \\
\text { Cumulative OR }{ }^{b}\left(95 \% \mathrm{Cl}^{c}\right)\end{array}$} & \multicolumn{2}{|c|}{$\begin{array}{l}\text { Physically } \\
\text { unhealthy days }\end{array}$} & \multicolumn{2}{|c|}{$\begin{array}{l}\text { Injury-caused } \\
\text { unhealthy days }\end{array}$} & \multicolumn{2}{|c|}{$\begin{array}{l}\text { Mentally unhealthy } \\
\text { days }{ }^{d}\end{array}$} \\
\hline & & $\beta$ & $P$ & $\beta$ & $P$ & $\beta$ & $P$ \\
\hline \multicolumn{8}{|l|}{ Age group (years) } \\
\hline $18-24$ & 1.00 & 0.00 & . & 0.00 & . & 0.00 & . \\
\hline $25-34$ & $1.36(1.22-1.50)$ & 0.04 & 0.63 & -0.06 & 0.24 & -0.03 & 0.63 \\
\hline $35-44$ & $1.83(1.62-2.07)$ & 0.32 & $<0.01$ & -0.08 & 0.26 & 0.02 & 0.82 \\
\hline $45-54$ & $2.71(2.37-3.10)$ & 0.81 & $<0.01$ & -0.01 & 0.86 & 0.10 & 0.21 \\
\hline $55-64$ & $3.58(3.10-4.13)$ & 1.17 & $<0.01$ & -0.01 & 0.85 & 0.11 & 0.20 \\
\hline $65-74$ & $4.62(3.93-5.44)$ & 1.76 & $<0.01$ & -0.03 & 0.68 & 0.07 & 0.47 \\
\hline$\geq 75$ & $4.88(4.05-5.88)$ & 1.78 & $<0.01$ & -0.03 & 0.73 & -0.06 & 0.49 \\
\hline \multicolumn{8}{|l|}{ Gender } \\
\hline Man & 1.00 & 0.00 & . & 0.00 & & 0.00 & . \\
\hline Woman & $1.25(1.19-1.31)$ & 0.47 & $<0.01$ & -0.03 & 0.06 & 0.22 & $<0.01$ \\
\hline \multicolumn{8}{|l|}{ Education } \\
\hline Illiterate or some primary school & 1.00 & 0.00 & . & 0.00 & . & 0.00 & \\
\hline Primary school graduate/some junior high school & $0.89(0.81-0.96)$ & -0.19 & 0.04 & 0.01 & 0.79 & 0.07 & 0.15 \\
\hline Junior high school graduate/some senior high school & $0.73(0.66-0.80)$ & -0.43 & $<0.01$ & -0.03 & 0.12 & -0.00 & 0.90 \\
\hline Senior high school graduate/some college & $0.73(0.64-0.83)$ & -0.29 & 0.01 & -0.06 & 0.03 & 0.17 & $<0.01$ \\
\hline College graduate or above & $0.68(0.56-0.82)$ & -0.24 & 0.04 & -0.05 & 0.07 & 0.45 & $<0.01$ \\
\hline \multicolumn{8}{|l|}{ Marital status } \\
\hline Single & $0.94(0.83-1.06)$ & 0.14 & 0.22 & 0.02 & 0.73 & -0.02 & 0.83 \\
\hline Married or cohabiting & $0.93(0.85-1.04)$ & -0.03 & 0.73 & 0.00 & 0.93 & -0.16 & $<0.01$ \\
\hline Separated/divorced/widowed/others & 1.00 & 0.00 & & 0.00 & . & 0.00 & \\
\hline \multicolumn{8}{|l|}{ Place of residence } \\
\hline Urban & $1.15(0.96-1.37)$ & 0.12 & 0.38 & -0.03 & 0.27 & 0.07 & 0.33 \\
\hline Rural & 1.00 & 0.00 & . & 0.00 & & 0.00 & . \\
\hline \multicolumn{8}{|l|}{ Geographic location } \\
\hline Eastern China & 1.00 & 0.00 & & 0.00 & & 0.00 & \\
\hline Central China & $1.42(1.15-1.75)$ & -0.05 & 0.70 & -0.03 & 0.07 & -0.00 & 0.93 \\
\hline Western China & $1.37(1.07-1.75)$ & 0.32 & 0.03 & 0.11 & 0.12 & 0.19 & 0.07 \\
\hline
\end{tabular}

${ }^{a}$ Examined the independent effects of covariates on SRH (very good, good, general, poor/very poor) by modeling a multiple ordered logistic regression

${ }^{b}$ Cumulative OR from an ordinal logistic regression model with adjustment for all covariates. Self-rated health (very good/good/general/poor/very poor) was the dependent variable. Each OR reflects the cumulative odds of rating poorer health versus better health against the cumulative odds in the reference group. Hence, the cumulative OR represents the average effect of the covariate on the cumulative odds of rating poorer health.

c The $95 \% \mathrm{Cls}$ take into account the complex survey design. It was no significantly difference between the covariate group and the reference group if 1 fall within $95 \% \mathrm{Cl}$ OR, and vice versa.

${ }^{d}$ Examined the independent effects of covariates on unhealthy days by multiple liner regression.

Source: Data from the 2010 China Chronic Disease and Risk Factor Surveillance survey

reported 0.17 and 0.45 more mentally unhealthy days than those with illiterate or some primary school, respectively.

\section{Discussion}

We used a large and nationally representative sample to examine for the first time the distribution of SRH and HRQOL status among Chinese adults in 2010. We found that the proportion of SRH was significantly different among age groups, gender groups, education groups and geographic location groups. Adults who were older, woman, lived in the western region, or had less education were more likely to report poorer SRH. Older adults reported more physically unhealthy days than younger ones. Women reported more physically unhealthy days and mentally unhealthy days than men. Adults with more education reported fewer physically unhealthy days and more mentally unhealthy days than those with less 
education. Adults in the west region reported more physically unhealthy days than those in the eastern region.

\section{SRH and HRQOL}

This study showed that $6.3 \%$ Chinese adults rated their health as poor or very poor, $47.9 \%$ good and $9.7 \%$ very good. Collectively, Chinese adults appear more positive in their ratings of health compared to many other countries.

The reported poor SRH status varied in different countries. Among adults aged 18 years old or above, the estimated overall rate of self-rated fair or poor (excellent, very good, good, fair and poor) health ranged from $10.1 \%$ in Minnesota to 30.9 \% in Puerto Rico [25], while $15.9 \%$ adults rated their health as fair or poor in U.S. in 2009 [26]. 9.7 \% Canadians reported fair or poor health in 2012 [27]. Approximately $15 \%$ of the Australian population rated their health as fair or poor in 2007 [28]. Only $1.5 \%$ Singaporeans rated their health as bad or very bad in 2001 [3]. The differences might be also partially due to the differences of the methodologies used for accessing SRH among different countries, such as the methods involved in eliciting a response, the questions phrased, and the methods used for interviewing.

A study showed the strong and nearly linear relationship between SRH status and the use of physician services during the following year, which mean the use of health services will increase with the SRH as poor or very poor [29]. Therefore, health care providers might use SRH to find persons with high risk of diseases and provide early treatment to reduce the cost of medical care.

We found that the mean number of physically unhealthy days was 1.48 , injury-caused unhealthy days 0.20 , and mentally unhealthy days 0.54 during the past 30 days in China. Therefore the physical illness remains a major problem for Chinese adults.

HRQOL can be influenced by participants' experiences, beliefs, expectations, and perceptions [21]. Mean numbers of physically and mentally unhealthy days were 3.6 and 3.5, respectively during the past 30 days in the U.S. in 2009 [22, 30]. Telephone interviews were used to collect data on SRH and HRQOL in BRFSS surveys in the U.S. while face-to-face interviews were conducted by gathering participants in certain central locations for the CCDRFS survey in China. Thus, adults who were severely ill or injured were not able to be interviewed. This may explain the potential reason why fewer physically unhealthy days and mentally unhealthy days were reported in China than those in U.S.

\section{Age}

Our study showed that older adults reported more physically unhealthy days than younger people as well as poorer SRH status. The mean number of physically unhealthy days was almost 3 days during the past 30 days and about $15 \%$ rated their health poor or very poor among those aged 75 and over. The poor health status of older people might be due to the increased chronic diseases. The reports from CCDRFS showed that $66.9 \%$ had hypertension, $19.6 \%$ had diabetes, $12.6 \%$ and 16.8 $\%$ self-reported incidences of myocardial infarction or stroke among the elderly aged 60 years or older in 2010 [31]. The population aged 60 and older reached 221 million in 2015, and now consists of $16 \%$ of the total Chinese population [31]. The population aging process will bring a significant impact on disease patterns and health status among Chinese. Our finding further suggests that poor health statuses in the elderly may be one of the main concerns for policy makers. The health care and medical services for the elderly population should be taken into account for implementing policies and interventions.

\section{Gender}

We found that women reported more physically unhealthy days and mentally unhealthy days than men as well as poorer SRH status. Similar findings were also reported in Singapore and the U.S. [3, 32]. The poorer health status of women is speculated to be caused by their physiological features, roles in society, numerous responsibilities, juggling work-life, and family duties. Furthermore, the previous study has shown that depression and anxiety disorders were more common in women than men [33]. Additionally, a U.S. study reported that U.S. women of lower social status were more likely to report poor health [34]. Thus, our finding indicates that more attention is needed for women's health and appropriate public health interventions should be implemented to improve women's health statuses in China. In addition, it would be beneficial to women's health if their social statuses are improved.

\section{Education}

In this study, we found that the physically unhealthy days declined with improving educational levels while mentally unhealthy days increased. Adults with higher education could typically obtain better jobs with higher incomes and better medical care, possibly leading to better physical status. However, adults with higher education might be subjected to higher expectations, possibly pressuring them into more mental health problems.

Our study also showed that the prevalence of selfreporting health as poorer was associated with less education. This finding was consistent with the reports from BRFSS [32], indicating that 1) strengthening national education is good for improving population health status and 2) increasing health education services and health 
promotion activities might improve the health status of the general Chinese population, and even more so in people with lower education levels.

\section{Place of residence and geographic location}

Our study showed adults in the west region and in the central region reported poorer health than those in the eastern region. The finding indicates that the substantial disparity in health status still exists across regions in China in 2010. SRH encompasses physical health, mental health, and functional capacity of persons [35] and is a proxy indicator for perceived burden of acute and chronic health conditions [6]. The finding of BRFSS in U.S. indicated substantial variations in fair or poor health at the state and local levels suggest differences in the underlying burden of chronic diseases, health-care coverage, and health behaviors among states and territories [25]. Therefore, our findings call for immediate public health intervention to eliminate the disparity across regions. Fortunately, one strategy to eliminate the health disparities across regions was proposed in "Healthy China 2020" Strategy Research Report in 2008 [36], asserting that more health policies and allocating resources for health should be applied to eliminate the health disparities across regions in China.

\section{Limitations}

There are several limitations in this study. First, the cross-sectional design does not allow for any inferences on causality. Second, although the-four-questions measuring SRH and HRQOL had good construct validity, predictive validity, reliability, and responsiveness [6], many respondents appeared to give a response that represented the overall impression of their health over the recent past versus an actual count of days. Therefore the data collected are susceptible to recall bias. Third, all participants were interviewed directly in certain central locations, thus the adults who were severely ill or injured were unable to be surveyed. Nonetheless, the CCDRFS is the largest nationwide Chinese survey and is a useful data source for evaluating the population health status. Thus, this study suggests that appropriate public health interventions are essential for improving health among different groups in the Chinese population.

\section{Conclusions}

Collectively, in this study, we assessed for the first time the status of SRH and HRQOL for adults from all provinces in China. Substantial variation exists in SRH and HRQOL status among age groups, gender groups, education groups, and regions. Taking these disparities into account is extremely important for identifying the health-related needs of vulnerable populations, developing health policy, and allocating resources.

\section{Abbreviations}

SRH: self-rated health; HRQOL: health-related quality of life; CDC: the Centers for Disease Control and Prevention; BRFSS: the population-based Behavioral Risk Factor Surveillance survey; NHS: National Health Survey; CCDRFS: China Chronic Disease and Risk Factor Surveillance; Cl: confidence interval; OR: odds ratios.

\section{Competing interests}

The authors declare that they have no competing interests.

\section{Authors' contributions}

LW, YL conceived and designed the survey. LW, YL, WD, and ZW performed the survey. YL, WD, and LQ analyzed the data. WD, YL, ZW, YJ, FM, LQ, JD, and LW contributed materials/analysis tools. WD, YL, ZW, YJ, FM, LQ, JD, and LW wrote the manuscript. All authors have read and approved the final version of this manuscript.

\section{Acknowledgements}

We thank all provincial and local health administrative departments, the Centres for Disease Control and Prevention, and all investigators for their support and hard work during the survey. We are also grateful for the valuable suggestions for the manuscript from Guang Zeng, Engelgau Michael, and Tao Shen from the Chinese Field Epidemiology Training Program. This work was supported by China's central government.

\section{Author details}

${ }^{1}$ National Center for Chronic and Noncommunicable Disease Control and Prevention, Beijing 100050, People's Republic of China. ${ }^{2}$ Chinese Field Epidemiology Training Program, Beijing 100050, People's Republic of China.

Received: 9 May 2015 Accepted: 6 January 2016

Published online: 12 January 2016

\section{References}

1. Bowling A. Health-related quality of life: Conceptral meaning, use and measurement. Buckingham: Open University Press; 2001.

2. Grad FP. The preamble of the constitution of the World Health Organization. Bulletin of the World Health Organization. 2002;80(12):981.

3. Lim W-Y, Ma S, Heng D, Bhalla V, Chew SK. Gender, ethnicity, health behaviour \& self-rated health in Singapore. BMC Public Health. 2007;7(1):184.

4. Månsson N-O, Råstam L. Self-rated health as a predictor of disability pension and death - a prospective study of middle-aged men. Scandinavian journal of public health. 2001;29(2):151-8.

5. DeSalvo KB, Fan VS, McDonell MB, Finn SD. Predicting mortality and healthcare utilization with a single question. Health services research. 2005; 40(4):1234-46.

6. Centers for Disease Control and Prevention, National center for chronic Disease Prevention and Health Promotion, Division of Adult and Community Health. Measuring healthy days: Population assessment of health-related quality of life. Atlanta: CDC 2000:4-6. http://www.cdc.gov/ hrqol/pdfs/mhd.pdf. Accessed 1 Jan 2015.

7. Gandek B, Sinclair SJ, Kosinski M, Ware Jr JE. Psychometric evaluation of the SF-36 health survey in Medicare managed care. Health Care Financing Review. 2004;25(4):5-25.

8. McHorney CA. Health status assessment methods for adults: Past accomplishments and future challenges. Annual review of public health. 1999;20(1):309-35

9. Selim AJ, Rogers W, Fleishman JA, Qian SX, Fincke BG, Rothendler JA, et al. Updated US population standard for the Veterans RAND 12-item Health Survey (VR-12). Quality of Life Research. 2009;18(1):43-52.

10. Kindig DA, Booske BC, Remington PL. Mobilizing Action Toward Community Health (MATCH): metrics, incentives, and partnerships for population health. Preventing chronic disease. 2010;7(4):A68.

11. Zack MM, Moriarty DG, Stroup DF, Ford ES, Mokdad AH. Worsening trends in adult health-related quality of life and self-rated health-United States, 1993-2001. Public health reports. 2004;119(5):493-505.

12. Cott CA, Gignac M, Badley EM. Determinants of self rated health for Canadians with chronic disease and disability. Journal of epidemiology and community health. 1999;53(11):731-6.

13. Shadbolt B. Some correlates of self-rated health for Australian women American Journal of Public Health. 1997;87(6):951-6. 
14. Delpierre C, Kelly-Irving M, Munch-Petersen M, Lauwers-Cances V, Datta GD, Lepage B, et al. SRH and HrQOL:does socialosition impact differently on their link with health status? BMC Public Health 2012;12:19

15. Yang G, Hu J, Rao KQ, Ma J, Rao C, Lopez AD. Mortality registration and surveillance in China: history, current situation and challenges. Popul Health Metr. 2005;3:3

16. Zhou M, Jiang Y, Huang Z, Wu F. Adjustment and representativeness evaluation of national disease surveillance points system. Ji Bing Jian Ce. 2010;25(3):239-44.

17. Zhao W, Ning G. National Workgroup of China Chronic Disease Surveillance. Methodology and content of China chronic disease surveillance (2010). Chin J Prev Med. 2012;46(5):477-9.

18. Chinese Center for Disease Control and Prevention,National Center for Chronic and Non-communicable Disease Control and Prevention. Report on chronic disease risk factor surveillance in China. Beijing: Military Medicalsciences publishing house; 2012.

19. Hennessy CH, Moriarty DG, Zack MM, Scherr PA, Brackbill R. Measuring health-related quality of life for public health surveillance. Public health reports. 1994;109(5):665-72.

20. Hu N, Jiang Y, Li Y, Chen Y. Weighted method of chronic disease risk factor suveilance in China, 2010. Chinese Journal of Health Statistics. 2012;29(3): 424-6.

21. Testa MA, Simonson DC. Assessment of quality-of-life outcomes. New England journal of medicine. 1996:334(13):835-40.

22. Centers for Disease Control and Prevention. Health-Related Quality of Life. Nationwide Trend. Mean physically unhealthy days: Overall. http://www.cdc. gov/hrqol/data/tables/table2a.htm. Accessed October 2015.

23. Selvin S. Statistical analysis of epidemiologic data. 2nd ed. New York: Oxford University Press; 1996.

24. Jing Y, Du Z, Jiang Y. Sampling technology. 2nd ed. Beijing: Renming university of China publishing house; 2008.

25. Li C, Balluz LS, Okoro CA, Strine TW, Lin JM, Town M, et al. Surveillance of certain health behaviors and conditions among states and selected local areas - behavioral risk factor surveillance system, United States, 2009. MMWR Surveill Summ. 2011;60(9):1-250.

26. Health-Related Quality of Life (HRQOL). Surveill and data. http://www.cdc. gov/hraol/data/tables/table1a.htm. Accessed 1 Jan 2015

27. Indicators of Well-being in Canada. Health - Self-rated Health. http://www4. hrsdc.gc.ca/.3ndic.1t.4r@-eng.jsp?iid=10\#M_1. Accessed 1 Jan 2015.

28. Mewton L, Andrews G. Poor self-rated health and its associations with somatisation in two Australian national surveys. BMJ Open. 2013;28:3(6).

29. Milunpalo S, Vuori I, Oja P, Pasanen M, Urpomen H. Self rated health status as a health measure: the predictive value of self reported health status on the use of physician services and on mortality in the working age population. Journal of Clinicle Epidemiology. 1997;50(5):517-28.

30. Centers for Disease Control and Prevention. Health-Related Quality of Life Nationwide Trend. Mean mentally unhealthy days: Overall. http://www.cdc. gov/hrqol/data/tables/table3a.htm. Accessed October 2015.

31. Nationa Centers for chronic and no-communication disease contrl and prevetion. Report on the status of elderly health:chronic disease risk factor surveillance in China(2010). Beijing: People's medical publishing house; 2014.

32. Centers for Disease Control and Prevention. National Center for Chronic Disease Prevention and Health Promotion. Health-Related Quality of Life. Nationwide Trend. Percentage with fair or poor self-rated health: Overall. http://www.cdc.gov/hrgol/data/tables/table1a.htm. Accessed October 2015.

33. World Health Organization: The World health report: 2001-Mental health: new understanding, new hope. 2001. http://www.who.int/whr/2001/en/. Accessed 1 Jan 2015

34. Jun H-J, Subramanian S, Gortmaker S, Kawachi I. A multilevel analysis of women's status and self-rated health in the United States. Journal of the American Medical Women's Association (1972). 2004:59(3):172-80.

35. Simon J, de Boer J, Joung I, Bosma H, Mackenbach J. How is your health in general? A qualitative study on self-assessed health. European Journal of Public Health. 2005;15(2):200-8

36. The editorial board of "Healthy China 2020" strategy research report. "Healthy China 2020" Strategy Research Report. Beinjing: People's medical publishing house; 2012.

\section{Submit your next manuscript to BioMed Central and we will help you at every step:}

- We accept pre-submission inquiries

- Our selector tool helps you to find the most relevant journal

- We provide round the clock customer support

- Convenient online submission

- Thorough peer review

- Inclusion in PubMed and all major indexing services

- Maximum visibility for your research

Submit your manuscript at www.biomedcentral.com/submit 